\title{
Hate Crime und/oder Hate Speech im Fadenkreuz des Strafrechts?
}

\author{
Kommentar zu Dharmapala/Garoupa/McAdams, Hate Crime: A Behavioral Analysis
}

Die Arbeit von Dharmapala, Garoupa und McAdams bietet eine faszinierende Analyse, die kognitive Psychologie und Ökonomie aufschlussreich auf rechtliche Probleme bezieht.

Meine Überlegungen und Bemerkungen werden gewiss auch durch den Umstand gefärbt, dass ich kein Strafrechtler bin; die beiden Gläser meiner Brille sind zum einen die Rechtsvergleichung und zum anderen das Äußerungsrecht, das zunehmend von den verfassungsrechtlichen Vorgaben der Meinungs- und Redefreiheit geprägt wird.

»Hate crimes«, d.h. Verbrechen gegen Personen, die als fremd und feindlich eingeschätzten Gruppierungen zugeordnet werden, sind leider zu einem nahezu universalen Phänomen geworden, auf das alle höher entwickelten Rechtssysteme reagiert haben. Die legislatorischen Antworten fallen freilich sehr unterschiedlich aus. Die USA - und ihnen folgend das Vereinigte Königsreich - haben »hate crime statutes« erlassen, die den Gegenstand der vorgetragenen Analyse bilden. Diese Gesetze sehen schärfere Sanktionen für Straftaten vor, die durch den Hass gegen Gruppen motiviert werden, die durch rassische oder ethnische Zuordnung, religiöse Überzeugungen, Geschlecht, Alter, sexuelle Orientierung oder andere Merkmale definiert werden. In den USA ist weiterhin sehr streitig, ob derartige Gesetze sinnvoll sind ${ }^{1}$. In anderen Ländern wird von solchen Gesetzen abgesehen. Dafür wird »hate speech«, die gegen die Angehörigen der bezeichneten oder anderer Gruppen gerichtete Hassrede und -propaganda mit Strafe bedroht ${ }^{2}$; in der Praxis geht es dabei fast durchweg um Äußerungen des Rassenhasses. Dieser Gesetzgebung haben Dharmapala und McAdams eine frühere Untersuchung gewidmet ${ }^{3}$. In ihr wird dargelegt, dass die Strafdrohung die Kosten für Rassenhetze steigert und deshalb das Volumen nicht nur von »hate speech«, sondern auch von »hate crime « zu reduzieren verspricht. In den USA werfen derartige Gesetze erhebliche verfassungsrechtliche Probleme auf: Sie gelten weithin als mit der Verbürgung der Redefreiheit unvereinbar ${ }^{4}$. Systematisch lassen sich drei Ansätze zur Bekämpfung der »hate crimes « unterscheiden:

1 Dafür etwa Harel/Parchomovsky, On Hate and Equality, 109 Yale L. J. 507 ff. (1999/ 2000); dagegen Hurd/Moore, Punishing Hatred and Prejudice, 56 Stanford L. Rev. $1081 \mathrm{ff}$. (2003/04).

2 Dazu näher Kübler, How much Freedom for Racist Speech?: Transnational Aspects of a Conflict of Human Rights, 27 Hofstra L. Rev. 335 ff. (1998), mwN.

3 Words that Kill? An Economic Model of the Influence of Speech on Behavior (with Particular Reference to Hate Speech), 34 J. of Legal Studies 93 ff. (2005).

4 R.A.V. v. City of St. Paul, 505 U.S. 377 (1992). 
1. Verbot der Verbreitung von Informationen über begangene »hate crimes«, da derartige Berichte als Hinweis auf schlechte Eigenschaften der Opfer verstanden werden können, die die Angriffe auf sie zu rechtfertigen vermögen und damit den Just World Bias (JWB) bestätigen. Die Arbeit erwähnt die Probleme dieses Ansatzes ${ }^{5}$ :

a) Das sind zum einen die »hohen Kosten«, und sie sind in der Tat prohibitiv: Medienberichte über »hate crimes « informieren über Ereignisse, an denen ein erhebliches und legitimes öffentliches Interesse besteht; soweit diese Berichte zutreffen, wäre jede Verbreitungsbeschränkung sowohl in den USA wie in Deutschland eindeutig verfassungswidrig; und sie verletzte zudem die Europäische Menschenrechtskonvention.

b) Zudem könnte sich das Verbot als kontraproduktiv erweisen. »Hate crimes « werden in den Medienberichten in aller Regel kritisiert und verurteilt; sie regen immer wieder zu Demonstrationen gegen Rassen- und Fremdenhass an und werden deshalb kaum den Eindruck vermitteln, diese Straftaten seien das verdiente Schicksal der Betroffenen.

2. Der amerikanische Ansatz: Strafverschärfung für durch Hass motivierte Straftaten. Er findet auf dem europäischen Kontinent wohl deshalb keine Nachahmung, weil das Strafrecht den Gerichten bei der Strafzumessung so große Spielräume belässt, dass die besonderen Umstände der Hassmotivation berücksichtigt werden können. Das ist anders in den USA: Dort wird dieses Ermessen durch die Fassung der Strafbestimmungen und durch die Sentencing Guidelines zunehmend eingeengt. Die Arbeit von Dharmapela, Garoupa und Mc Adams überzeugt auch durch die Trennung von zwei Tatzeiträumen und durch den Nachweis, wie die in der Periode 1 begangenen Straftaten das Ausmaß der Straftaten in Periode 2 beeinflussen. Hier drängt sich freilich die Frage auf, wie realistisch die Annahme ist, dass die Verschärfung der angedrohten Strafen die Kriminalität in Periode 1 effektiv vermindern wird; ich sehe mehrere Gründe, dies zu bezweifeln:

a) Der traditionelle ökonomische Ansatz operiert mit einer Kosten-Nutzen-Analyse: Die Abschreckung wirkt, wenn die angedrohte Strafe den von der Straftat erwarteten Nutzen überwiegt. Die Verhaltensforschung gibt einigen Anlass, an dieser Erklärung zu zweifeln. Der Vorteil aus dem Rechtsverstoß wird sofort gezogen, die Kosten der eventuellen Bestrafung fallen - wenn überhaupt - erst in der Zukunft an; deshalb werden diese künftigen Kosten abgezinst. Das heißt: Die Strafdrohung an sich mag durchaus wirksam sein; eine Erhöhung von fünf auf sechs Jahre Haft wird den potentiellen Täter aber kaum beeindrucken ${ }^{6}$. Hinzutreten können der vielfach belegte übermäßige Optimismus, einschließlich der Überschätzung der eigenen Fähigkeiten (etwa: sich der Entdeckung und Bestrafung zu entziehen $)^{7}$. Dem kann durch Intensivierung der polizeilichen Ermitt-

5 Dharmapala/Garoupa/McAdams S. $126 \mathrm{f}$.

6 Dazu Jolls/Sunstein/Thaler, A Behavioral Approach to Law and Economics, 50 Stanford L. Rev. 1471, 1538 f. (1997/98) mwN.

7 Dazu etwa Taylor, Positive Illusions (1989) S. 10 ff. und 39 f.; Camerer/Lovallo, Overconfidence and Excess Entry: An Experimental Approach, 89 Am Econ. Rev. 306 (1999). 
lungs- und Verfolgungsbemühungen entgegengewirkt werden. Das ist aber speziell für »hate crimes « nicht möglich, da sich die Motivation der Täter in aller Regel erst im Laufe der Ermittlungen feststellen lässt ${ }^{8}$.

b) Hinzu kommen empirische Befunde, die auf der Makro-Ebene erhoben worden sind. Im Verhältnis zur Gesamtbevölkerung ist die Zahl der Strafhäftlinge in den USA mehr als zehn mal so hoch wie in Dänemark ${ }^{9}$; und in Dänemark wird die Todesstrafe weder verhängt noch vollstreckt. Das heißt: In den USA ist die Kriminalität sehr viel höher als in Dänemark, obwohl die angedrohten und verhängten Strafen nach europäischen Maßstäben drakonisch sind; das erweckt nicht den Eindruck wirksamer Abschreckung.

c) Schließlich ist zu fragen, ob die Abschreckungswirkung nicht auch von der Art der Straftat abhängt. Sie dürfte relativ hoch sein im Bereich der Wirtschaftskriminalität; dort werden Kosten-Nutzen-Abwägungen keine Ausnahme sein. Anders könnte oder dürfte es sich dort verhalten, wo die Straftat nicht durch Gewinnstreben, sondern emotional motiviert wird; und das ist bei »hate crimes« offensichtlich der Fall.

3. Damit komme ich zu der europäischen Reaktion, d.h. zu einer Gesetzgebung, die die Verhetzung, die Aufstachelung zu Hass und Gewalt gegen Teile der Bevölkerung, mit Strafe bedroht. Die erwähnte Untersuchung aus dem Jahr $2005^{10} \mathrm{kommt}$ zu dem Ergebnis, dass derartige Gesetze die Kosten für »hate speech« erhöhen und damit das Ausmaß von »hate crimes « reduzieren. Das wird damit begründet, dass »hate speech« den Nutzen erhöht, den sich der Täter von der Ausübung eines »hate crimes « verspricht: Er maximiert das Ansehen, »the esteem«, das er bei Gleichgesinnten genießt; das wird aus der »esteem theory« abgeleitet. D.h.: Die Hasspropaganda vermittelt die Information, dass sich durch die Gewaltanwendung die Achtung und Zustimmung im eigenen sozialen Umfeld mehren lässt.

4. An dieser Stelle drängt sich die Frage auf, ob sich der Ansatz des »Just World Bias« nicht auch auf die Gesetze anwenden lässt, die »hate speech« verbieten. Hasspropaganda zielt darauf ab, den Glauben daran zu vertiefen, dass es sich bei der angeprangerten Gruppe um Personen mit so negativen Merkmalen handelt, dass ihre Behelligung und Verfolgung nicht nur legitim, sondern geradezu geboten ist. Dadurch wird wiederum der Glaube an die Gerechtigkeit der bestehenden Zustände gefestigt: Die Opfer verdienen, was ihnen angetan wird; sie haben sich ihr Schicksal selber zuzuschreiben.

Auch diese Überlegung vermag den Eindruck zu stärken, dass es rechtspolitisch sinnvoller und wirksamer ist, »hate speech« zu verbieten und $\mathrm{zu}$ bestrafen, als die Strafdrohung für solche Taten zu verschärfen, die durch Hass motiviert worden sind.

8 Dazu Hurd/Moore (FN 1) S. 1139.

9 Diese und andere Zahlen bei Whitman, Harsh Justice. Criminal Punishment and the Widening Divide between America and Europe (2003) S. 57 f.

10 Vgl. oben zu FN 3. 